Research Article

\title{
Denoising of Tourist Street Scene Image Based on ROF Model of Second-Order Partial Differential Equation
}

\author{
Xiaofeng Yang $(\mathbb{D}$ \\ School of Humanities and Education, Xijing University, Xi'an, 710123 Shaanxi, China \\ Correspondence should be addressed to Xiaofeng Yang; 20070063@xijing.edu.cn
}

Received 17 September 2021; Accepted 22 October 2021; Published 31 October 2021

Academic Editor: Miaochao Chen

Copyright (C) 2021 Xiaofeng Yang. This is an open access article distributed under the Creative Commons Attribution License, which permits unrestricted use, distribution, and reproduction in any medium, provided the original work is properly cited.

\begin{abstract}
The noise pollution in tourist street view images is caused by various reasons. A major challenge that researchers have been facing is to find a way to effectively remove noise. Although in the past few decades people have proposed many methods of denoising tourist street scene images, the research on denoising technology of tourist street scene images is still not outdated. There is no doubt that it has become a basic and important research topic in the field of digital image processing. The evolutionary diffusion method based on partial differential equations is helpful to improve the quality of noisy tourist street scene images. This method can process tourist street scene images according to people's expected diffusion behavior. The adaptive total variation model proposed in this paper is improved on the basis of the total variation model and the Gaussian thermal diffusion model. We analyze the classic variational PDE-based denoising model and get a unified variational PDE energy functional model. We also give a detailed analysis of the diffusion performance of the total variational model and then propose an adaptive total variational diffusion model. By improving the diffusion coefficient and introducing a curvature operator that can distinguish details such as edges, it can effectively denoise the tourist street scene image, and it also has a good effect on avoiding the step effect. Through the improvement of the ROF model, the loyalty term and regular term of the model are parameterized, the adaptive total variation denoising model of this paper is established, and a detailed analysis is carried out. The experimental results show that compared with some traditional denoising models, the model in this paper can effectively suppress the step effect in the denoising process, while protecting the texture details of the edge area of the tourist street scene image. In addition, the model in this paper is superior to traditional denoising models in terms of denoising performance and texture structure protection.
\end{abstract}

\section{Introduction}

Visual information accounts for up to $60 \%$ of all kinds of information. Therefore, tourist street view image information is the main source of all kinds of information that humans come into contact with. During the acquisition or transmission of tourist street view images, due to imaging principles, sensor defects, and changes in the surrounding environment, the signal may be unstable, and noises of different intensities may be generated, resulting in degradation of the tourist street view image quality [1]. Noise can be understood as interference signals that affect the acquisition or analysis of tourist street scene images from the signal source received by people or sensors. Noise can be divided into additive noise and multiplicative noise. On the one hand, noise affects the visual effect of tourist street scene images, and at the same time, it affects people's normal visual judgment and seriously interferes with the accurate acquisition of tourist street scene image information [2]. Denoising of tourist street scene images is a very important early stage of tourist street scene image processing. Many tourist street scene image processing tasks, such as tourist street scene image segmentation and edge extraction, all depend on the effect of denoising [3]. Therefore, how to better remove noise is a more critical part in the preprocessing of tourist street view images. In recent years, the PDE-based tourist street view 
image processing method has become a new type of tourist street view image processing mathematical tool due to its formal standardization, model building flexibility, and local adaptive characteristics, and it is widely used in tourism [4]. Another notable feature is the composability of different algorithms. Finally, an important advantage of the PDEbased method is that with the help of numerical analysis, the partial differential equation method can obtain higher stability and accuracy.

The noise of tourist street view images is a variety of factors that hinder people from accepting its information in tourist street view images. According to the interference of noise to tourist street view image information, noise is usually divided into two categories: additive noise and multiplicative noise. Under normal circumstances, the signal embodied by noise is random and irregular, which is also unexpected, but its existence will affect the entire process of tourist street view image processing, especially in the stage of tourist street view image acquisition [5]. Therefore, the removal of noise plays a huge role in the image processing of tourist street scenes. On the one hand, this process needs to be applied in the preprocessing stage of tourist street view image processing. This process is also the intersection of tourist street view image restoration and tourist street view image enhancement. The denoising method of tourist street view image refers to removing noise factors from the existing noisy tourist street view image with the help of various filtering models. The to-beprocessed noisy tourist street view image mainly covers many different elements such as edges, textures, sharp corners, and noise. The denoising of tourist street view image is to use various mathematical tools to establish appropriate mathematical models. The tourist street view image information and noise information are processed more effectively [6]. During the transmission of tourist street scene images, noise will also be generated due to the nature of the transmission medium; in the storage of tourist street scene images, due to the realization of the storage algorithm, noise will also be generated, which will reduce the quality of tourist street scene images [7]. However, in practical applications, a tourist street view image with a certain definition standard is needed. Therefore, in order to improve the quality of tourist street scene images, denoising of tourist street scene images has very important practical significance. In fact, denoising of tourist street scene images is the most basic problem in the field of tourist street scene image processing. It also has important theoretical significance for the later tourist street scene image understanding and tourist street scene image analysis in tourist street scene image processing.

We use the variational method of partial differential equation (PDE) to process tourist street scene image. Modeling is based on the priori model of the tourist street view image, and then, the processing problem of the tourist street view image is transformed into a functional extremum problem, and the Euler-Lagrange equation is derived to solve it. In this article, we briefly introduce the methods used in the process of processing tourist street scene images with variational partial differential equations. Through the analysis of the classic variational PDE-based denoising model, a unified variational PDE energy functional model is obtained; by improving the total variation denoising model, the loyalty term and regular term of the model are parameterized and established, which greatly improves the denoising effect. The improved PM diffusion model can split and control the diffusion direction and suppress the diffusion in the gradient direction, thereby protecting the edge information of the tourist street scene image. According to the experimental results, the model in this paper can protect the edge information and structural features of tourist street scene images during the denoising process and improve the performance and stability of denoising. A denoising model for tourist street scene images that combines improved PM and recursive filtering is proposed. This model can not only suppress the diffusion in the gradient direction and protect the structural information of the edge area of tourist street scene images but also update the pixels in a recursive manner and reduce the number of iterations and retain the texture information of the tourist street view image. From the experimental results, the model in this paper can take into account the edge structure features and texture details of the tourist street view image when eliminating noise. Compared with the traditional model, the denoising ability is stronger, and the obtained tourist street view image has better visibility.

\section{Related Work}

In the field of computer vision and tourist street view image processing, PDE-based denoising is a new field that has grown rapidly after nearly two decades. The problems of tourist street scene image denoising, tourist street scene image restoration, and tourist street scene image segmentation in tourist street scene image processing have attracted extensive research from a large number of tourist street scene image processing enthusiasts and set off an upsurge in processing tourist street scene images using the PDE method. Now, this field has accumulated a lot of research results, and its unstoppable development trend has seriously impacted the traditional tourist street view image processing methods. Denoising has always been one of the hot issues in the field of tourist street view image processing. The PDE-based denoising method for tourist street scene images can not only effectively eliminate the noise of tourist street scene images but also retain some important details such as edge information and texture. Therefore, the proposal of this method has attracted wide attention from researchers at home and abroad.

There are two types of commonly used modeling methods. One is to establish the energy functional of the model and use the variational method to obtain the EulerLagrange equation of the model. This equation is the PDE needed to solve the model. The second is to rely on a certain physical process to achieve the desired tourist street view image change process and then establish the corresponding PDE. When the model is established, the problem of solving PDE becomes the primary consideration.

Related scholars have proposed a new type of nonlocal mean denoising model [8]. The denoising principle of this model is mainly to use the self-similarity between the pixels in the tourist street view image, and the average gray value of each pixel is calculated to suppress noise. Nonlocal mean 
denoising is not a tourist street view. The individual pixel values in the image are compared. On the contrary, this model is mainly aimed at comparing the gray value in the neighborhood of the pixel with this pixel as the center and generating weights based on the similarity of the gray distribution. Therefore, the repaired model of the nonlocal mean denoising model is visually satisfactory. Based on the ROF model, related scholars introduced the logarithm to the fidelity term, proposed a new Poisson denoising model (LCA model) based on the total variation regularity, and used an explicit forward difference scheme [9]. In the gradient descent flow equation, the proposed method has the advantages of eliminating noises of different scales and maintaining the lowcontrast characteristics of low-intensity regions. However, this method has two shortcomings: one shortcoming is that the denoised tourist street view image cannot be guaranteed to be positive, so it is meaningless to take the logarithm of it; the other shortcoming is that the time step must be taken due to the limited time step of the CFL condition.

There are two common methods for noise removal: one is to denoise in the spatial domain; the other is to denoise in the frequency domain. Denoising in the spatial domain, that is, spatial filters are mainly divided into linear and nonlinear spatial filtering. Linear spatial filters can be divided into high-pass filters, low-pass filters (typically represented by linear average filters), and band-pass filters, while nonlinear spatial filters mainly include median filtering, maximum and minimum filtering, and adaptive filtering. With the further exploration of the researchers where they found that this kind of initial filter is increasingly unable to meet the effect of more accurate noise removal, the corresponding wavelet transform based on multiscale analysis and time-frequency localization characteristics is proposed [10]. This type of transformation can not only remove noise very well but also play an important role in the processing and feature extraction of edge signals and peak mutations. Prior to this, the theoretical framework of partial differential equations was applied to the image processing of tourist street scenes. Thoughts have already been put forward. So far, the development of partial differential equation denoising theory has reached a certain height and has become one of the research hotspots in today's society [11-13].

At present, fractional calculus is in a stage of rapid development in many fields of science and engineering. Some improved fractional calculus processing algorithms are widely used in the image processing of tourist street scenes $[14,15]$. For the enhancement of tourist street view images, related scholars have proposed a new medical tourist street view image enhancement method, which adaptively adjusts the fractional order according to the dynamic gradient of the entire tourist street view image [16]. Researchers have proposed an adaptive tourist street view image enhancement algorithm that can automatically generate fractional differential orders based on the mask window size, tourist street view image gradient, and other theories [17]. According to the local statistical information and structural characteristics of tourist street scene images, the order of fractional differential is dynamically adjusted. Related scholars have proposed a variable-order fractional differential tourist street scene image enhancement algorithm [18]. Related scholars have proposed a differential curvature-driven fractional anisotropic diffusion of tourist street scene image denoising model, using two new technologies, fractional differential and differential curvature, to describe the intensity changes of tourist street scene images [19]. Researchers apply the fractional Brownian motion model and the regularization method of diffusion to anisotropy to propose a denoising algorithm for tourist street scene images containing natural random textures [20].

\section{Key Technology Analysis of Denoising of Tourist Street Scene Images}

3.1. Representation of Digital Tourist Street View Images. Usually, we can use a two-dimensional continuous function $I(x, y)$ to represent a tourist street view image in nature, where $(x, y)$ represents a pair of coordinate points in a twodimensional space, and $I(x, y)$ represents the gray value of the tourist street view image at this point. In order to facilitate computer processing, it is necessary to transform the continuous tourist street view image into a digital tourist street view image by sampling and quantizing the continuous tourist street view image. Sampling and quantization here mean that the coordinate value and the amplitude are, respectively, digitally processed. In actual application, the tourist street view image we see is obtained through various sensing devices, and the sampling method is determined by the sensor configuration that generates the tourist street view image.

Suppose an $M \times N$ two-dimensional array is used to represent the tourist street view image $u(x, y)$ sampled at equal intervals. The array contains $M$ rows and $N$ columns, where $(x, y)$ are discrete coordinates; usually, $x=0,1,2, \cdots, M-1$ and $y=0,1,2, \cdots, N-1$ represent discrete coordinates. Each point in the matrix is called a pixel point.

3.2. Noise Model of Tourist Street Scene Images. Tourist street scene image noise is an uncertain random signal generated due to various interferences when collecting or transmitting tourist street scene images. According to the classification of noise sources, it can be divided into internal noise interference and external noise interference. Generally, the interference caused by the influence of the components in the tourist street view image acquisition equipment can be defined as internal noise, such as the thermal movement of free electrons in the internal electronic circuit of the imaging equipment and the fluctuation of semiconductor carriers. Disturbance in the form of electromagnetic waves, such as electrical equipment and the discharge phenomenon of thunderstorms, is called external noise. The process of denoising tourist street scene images is shown in Figure 1.

According to the correlation between the noise signal and the tourist street view image signal itself, the noise can be divided into additive noise and multiplicative noise. Additive noise refers to the existence of noise regardless of whether the tourist street view image signal exists or not, so additive noise is often regarded as the background noise of the system. For example, when we use an imaging device composed of a charge-coupled device sensor to obtain a tourist street view image, the noise caused by the influence 


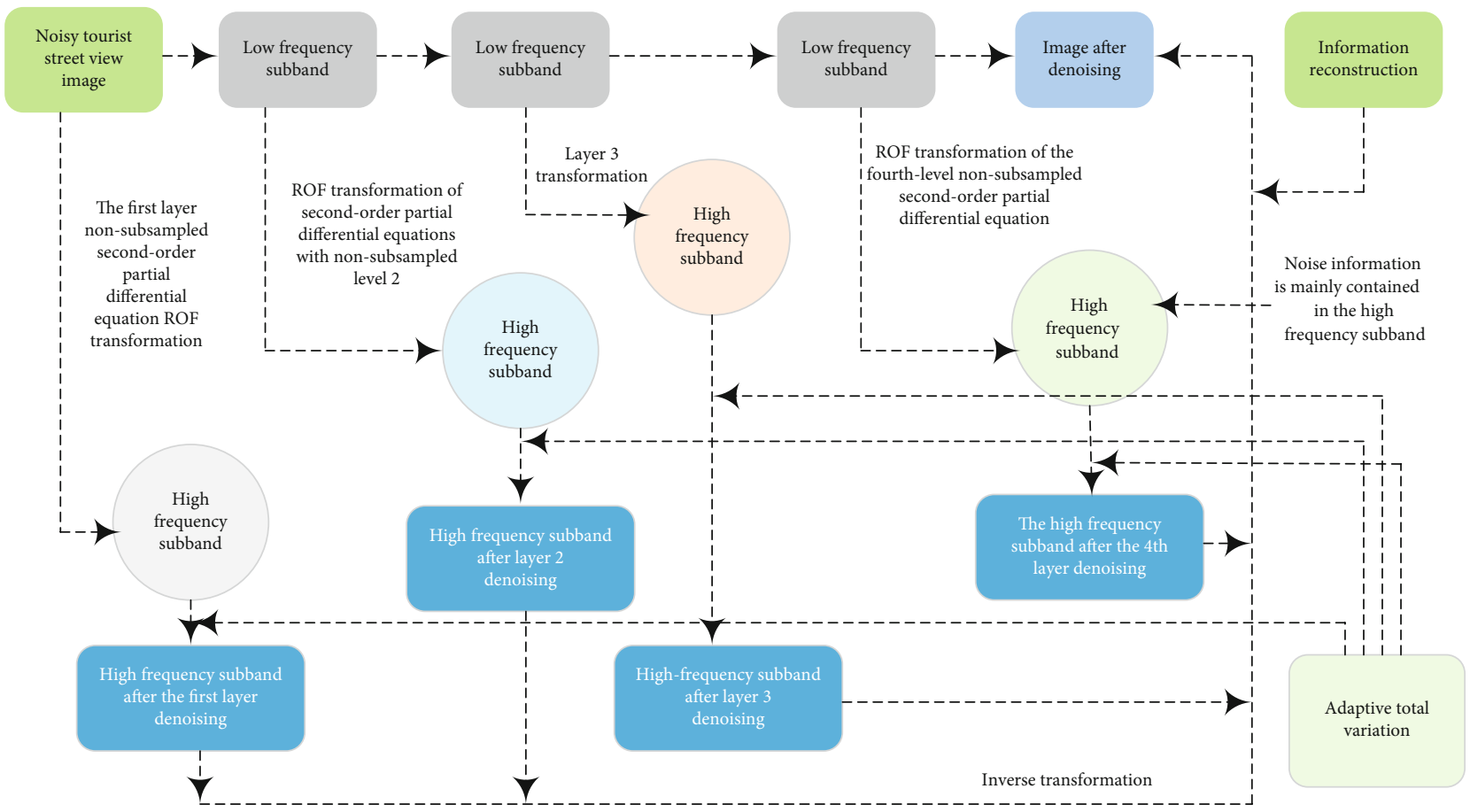

FIgURE 1: The process of denoising tourist street scene images.

of the light level and the temperature of the sensor belongs to this type of noise. Multiplicative noise is mainly caused by irregular changes in channel characteristics during signal transmission, such as noise in common synthetic aperture radar imaging. After the electromagnetic wave emitted by the radar encounters the rough surface of the object to be measured and is reflected, the rough surface contains multiple reflectors, which causes the reflected waves to be no longer coherent in phase, resulting in the lack of amplitude and intensity of the echo received by the radar. It presents coherent speckle noise, which can be classified as multiplicative noise. This article mainly studies how to remove the additive noise in tourist street scene images, because the noise in tourist street scene images that people usually encounter is additive noise formed by external interference.

The degraded tourist street view image with additive noise can be represented by the following model:

$$
u_{0}(x, y)=u(x, y)+f(x, y) \text {. }
$$

Among them, $u(x, y)$ represents the pollution-free tourist street view image, $u_{0}(x, y)$ represents the observed noisy tourist street view image, and $f(x, y)$ represents the noise interference.

We often use Gaussian noise in our research to simulate the unknown real noise in the environment. That is because firstly simulating noise is easy to objectively evaluate the effect of the algorithm. If we experiment with real noisy tourist street view images, we cannot know what the pollution-free tourist street view image corresponding to real noise looks like, so we can only rely on some subjective evaluations. And often, different people may have different preferences for tourist street view images, which will lead to too much individual difference in the final evaluation results; secondly, because there may be multiple noise sources in the real world, if we combine various noises, the source is regarded as mutually independent random variables. According to the central limit theorem, the mean value of a large number of mutually independent random variables will eventually converge to a normal distribution. The probability density function of Gaussian random variable $x$ is expressed as follows:

$$
p(x)=\sqrt{2 \pi} \sigma e^{-2 \sigma^{2} \cdot(u-x)^{2}} .
$$

Among them, $x$ represents the gray value of the tourist street view image, $\mu$ represents the mean value, and $\sigma$ represents the standard deviation. The greater the value of $\sigma$ is, the greater the noise intensity is.

3.3. Objective Evaluation Method for Denoising of Tourist Street View Images. When performing numerical experiments to verify the effect of the algorithm, the quality of the processing results can be evaluated through subjective evaluation and objective evaluation. Subjective evaluation mainly uses human eyes to observe the edge retention of tourist street view images, whether the flat area is smooth, etc. However, different people often have different views on things, so objective evaluation indicators are often used in research to evaluate an algorithm. Commonly used objective evaluation indicators include peak signal-to-noise ratio (PSNR) and average structural similarity measure (MSSIM).

Assuming that a digital tourist street view image is composed of $M \times N$ pixels, the PSNR is defined as follows:

$$
\operatorname{PSNR}=-2 \ln \left[M N\left(u_{x, y}-I_{x, y}\right)\right] .
$$


Among them, $I_{x, y}$ represents a reference tourist street view image without noise, and $u_{x, y}$ represents a tourist street view image after denoising. MSSIM is defined as follows:

$$
\begin{gathered}
\operatorname{SSIM}\left(I_{1}, I_{2}\right)=\frac{\left(c_{1}+u_{I 1} u_{I 2}\right)\left(2 c_{2}+\sigma_{I 1 I 2}\right)}{\left(c_{1}+u_{I 1}{ }^{2} u_{I 2}{ }^{2}\right)\left(2 c_{2}+\sigma_{I 1 I 2}^{2}\right)}, \\
\operatorname{MSSIM}\left(I_{2}, I_{2}\right)=\frac{u_{I 1} u_{I 2}}{W\left(u_{I 1}+u_{I 2}\right)} \prod_{i=0}^{W-1} \operatorname{SSIM}\left(x_{i}, 2 y_{i}\right) .
\end{gathered}
$$

In the formula, $u_{I 1}$ and $u_{I 2}$ represent the average value of tourist street view image $I_{j}$, and $\sigma_{2}$ represents the variance of tourist street view image $I_{1}$ and $I_{2} \cdot c_{1}$ and $c_{2}$ are very small constants; they are mainly used to avoid the occurrence of zero denominator; $W$ represents the number of partial windows divided by the tourist street view image $I_{j} ; x_{i}$ and $y_{i}$, respectively, represent the $i$ th partial window of the tourist street view image $I_{j}$.

\section{Adaptive Full Variational Denoising Model Based on Second-Order Partial Differential Equations}

4.1. Euler-Lagrange Equation and Gradient Descent Flow. The process of variational method of seeking extreme values of functionals can be understood as the process of solving partial differential equations [21-23]. Below, we will explore the EulerLagrange equation of the variational problem. For the energy functional in one-dimensional case, it can be expressed as

$$
E(u)=u \int_{x_{0}}^{x_{1}} F\left(-x, u,-u_{x}\right) f(x) d x
$$

And the function $u(x)$ satisfies the end point fixed condition. Our goal is to minimize the energy functional $E(u)$. In the knowledge of calculus, the corresponding extreme point of $f(x)$ should be the point where the derivative is 0. Similarly, the extreme value of the functional $E(u)$ should be the function corresponding to the strain point.

We make a perturbation $v(x)$ on $u(x)$. When $v(x)$ and $v(x)^{\prime}$ are small enough, Taylor gives

$$
F\left(x, u+v^{\prime}, u^{\prime}-v\right)=F\left(x, v^{\prime},-v\right)+\frac{\partial F}{\partial v} u-\frac{\partial F}{\partial v^{\prime}} u^{\prime} .
$$

Therefore, we get the following equation:

$$
E(u-v)=E(-u)+\int_{x_{0}}^{x_{1}}\left(u^{\prime} \frac{\partial F}{\partial v}-\frac{\partial F}{\partial u^{\prime}} v\right) d x
$$

According to the point method,

$$
\int_{x_{0}}^{x_{1}} \frac{\partial F}{\partial v} u d x=-u \frac{\partial F}{\partial v^{\prime}} \int_{x_{0}}^{x_{1}} \frac{\partial F}{\partial u^{\prime}} v^{\prime} d x
$$

When the energy functional $E(u)$ reaches its extreme value, we apply any perturbation $v(x)$ to $u(x)$, and its value remains unchanged, and the function of the following equation is obtained.

$$
\frac{\partial F}{\partial u}=-\frac{d}{d x}\left(\frac{\partial F}{\partial v^{\prime}}\right)
$$

Similar to the multivariate high-order functional extremum problem composed of multiple unknowns, the EulerLagrange equation can also be written using the above method. In order to solve the Euler-Lagrange equation, it is necessary to introduce a "time" auxiliary variable, so as to convert the static nonlinear partial differential equation problem into a dynamic problem. When the evolution problem tends to a steady state, the Euler-Lagrange equation solution of the variational problem is obtained. This is the gradient descent flow method.

In relatively simple cases, we can directly obtain the solution by solving the partial differential equation. However, most cases of denoising tourist street scene images are relatively nonlinear. Solving the PDE directly requires a large amount of numerical calculation and is very difficult. One of the relatively easy methods is the gradient descent method. The basic idea of the gradient descent method is to start from a given trial function, search in the direction of the fastest decline of the functional value, and finally reach the local minimum point. The gradient descent method is also called the steepest descent method, which is an optimization algorithm for searching for the extreme value of the function $[24,25]$. In the one-dimensional case, introducing the time variable $t$ into the Euler-Lagrange equation only needs to satisfy the following formula to make the energy value of the functional decrease continuously with the change of time:

$$
\frac{\partial u}{\partial t}=\frac{d}{d x}\left(\frac{\partial F}{\partial v}\right)+\frac{\partial F}{\partial v^{\prime}}
$$

4.2. Diffusion Analysis of Total Variation Model. The total variation model is a relatively successful model in the field of denoising. The classic total variation denoising model is obtained by improving the well-known ROF model. Similarly, we also analyze the principle of the total variation model by analyzing the performance of the ROF model. The energy function defined by the ROF model is

$$
E(u)=\iint_{D}|\nabla u| d x d y
$$

We use the steepest descent method to solve and get

$$
\frac{\partial u}{\partial t}=\nabla\left|\frac{1+\nabla u}{1-\nabla u}\right|
$$

This item is called the regular item or diffusion item, and it is also the amount of change of the tourist street view image each time it is updated. It will diffuse the tourist street view image information such as pixels in the noisy area to the contaminated area. In this way, as the iterative operation 
proceeds, when the diffusion term tends to a steady state, the minimization value is easily obtained. In order to avoid the value of the gradient in the diffusion term being zero and making the equation unsolvable, we generally set a tiny positive number $\varepsilon$, so that the gradient value is

$$
\nabla u_{\varepsilon}=\left[\nabla u^{2}-\varepsilon(u-v)\right]^{1 / 2}
$$

This paper uses $(\xi, \eta)$ coordinates to represent the diffusion equation. The tangent direction of the isoilluminance line at the local area point is represented by the $\xi$ axis, the $\eta$ axis is the gradient direction of the tourist street view image at the $(x, y)$ point, and the $(x, y)$ coordinates are only for the position of the pixel point of the tourist street view image. And $\nabla(\xi, \eta)$ coordinates represent the important information of the isoilluminance line of the tourist street scene image.

Through the performance analysis of the diffusion term of the total variation model, it can be seen that the biggest feature of the total variation model denoising is that it can well protect the edge of the tourist street scene image in the process of denoising. Because the diffusion is only along the direction of the tangent to the isoline, it does not diffuse along the gradient direction. There are two tangential directions and gradient directions at any point in a general tourist street view image. And if it encounters a smooth area of the tourist street view image, the model still spreads according to the edge direction; then, the final result will lead to false edges in the tourist street view image. Because there is no contour edge in the smooth area of the tourist street view image, this is also the typical defect of the total variation model-the step effect. So how to avoid the step effect and effectively denoise the image of tourist street scene is the focus of the improved model in this paper.

\subsection{The Establishment of an Adaptive Total Variational} Model of Second-Order Partial Differential Equations. The parameter $p$ represents the influence of the diffusion model on the smoothness of the tourist street view image. The larger the $p$, the smoother the tourist street view image, and the smaller the value, the stronger the step effect of the tourist street view image. Therefore, how to set the value of $\mathrm{p}$ according to the inherent characteristics of tourist street view images is also one of the key issues. The flow of the adaptive full variational algorithm for second-order partial differential equations is shown in Figure 2.

We use the second-order partial derivative of the difference curvature as the edge detection operator of the regional features of the tourist street scene image. The traditional edge detection operator based on a gradient cannot effectively distinguish the edge area and the slope area. Therefore, the differential curvature based on the second derivative can be effectively expressed as

$$
d=\left|u^{\prime} u_{\eta}\right|-\left|v^{\prime} u_{\xi}\right|
$$

The extraction effect using the second-order difference curvature operator is much better than that of the one-step operator. Adding the edge extraction function of the differential curvature operator to our model can make our model have the ability to distinguish the edge of the tourist street view image from the slope area, and it can also enhance the denoising function. Then, there are

$$
\begin{gathered}
J_{r}(u)=\int_{\Omega} r(\nabla u) p^{d} \lambda_{D} d x d y-\int_{\Omega}\left(\nabla u-u_{0}\right) d x d y, \\
\lambda_{D}(x)= \begin{cases}-1 & x \longrightarrow D \\
x u^{\prime}-2 v^{\prime} & x \longrightarrow \frac{\Omega}{2 D}\end{cases}
\end{gathered}
$$

The adaptive total variation model is based on the ROF model and achieves higher tourist street view image quality by adjusting the parameters of the regular term and the loyalty term. In the improved method of this paper, we choose to use a smaller $\varepsilon$ parameter in the regular term and choose a larger $\varepsilon$ parameter to apply to the loyalty term. In this way, choosing a larger $\varepsilon$ for the Lagrange multiplier in the loyalty term will greatly ease the smoothness. The regular term can not only effectively denoise but also protect the edges. The following is a parameter analysis of the regular term and loyalty term of the model.

Introducing the regular term parameter $\varepsilon$, where $\varepsilon>0$, the adaptive full variational model can be written as

$$
J_{s}(u)=\int_{D} \varepsilon v^{\prime} \lambda_{D} \nabla u(-x)-\int_{D}\left[u(x)-u_{0}(-x)\right]^{2} d x .
$$

$\lambda$ is the Lagrange multiplier and also the loyalty parameter of the model. The solution of the Euler-Lagrange equation corresponding to the above formula is

$$
\nabla u|\nabla u-\varepsilon|+\lambda_{D}\left(u_{0}-v^{\prime}\right)=0
$$

\section{Simulation Experiment and Analysis}

5.1. Anisotropic Diffusion Experiment. In order to verify the rationality and feasibility of the model algorithm in this paper, Matlab software is used for experimental simulation analysis, and the performance and benefit of the model are evaluated through the mean square error (MSE), peak signal-to-noise ratio (PSNR), and structural similarity (SSIM). The experiment uses a tourist street view image with a pixel size of 512 $\times 512$. We add Gaussian white noise with a variance of 20 to it and then perform simulation analysis.

For the noise-added tourist street view images, PM model, YK model, TV model, WHT model, and the model of this paper are used to denoise. The time step of each model is 3, and the coefficients of this model are set. The Canny operator is used to extract the edge feature of the noisy tourist street scene image and the denoising result image of each model, respectively. The denoising effect of the tourist street view image processed by the model in this paper is better, and the edge features are more complete. This is because the model in this paper splits the diffusion process and suppresses the gradient direction in the edge 


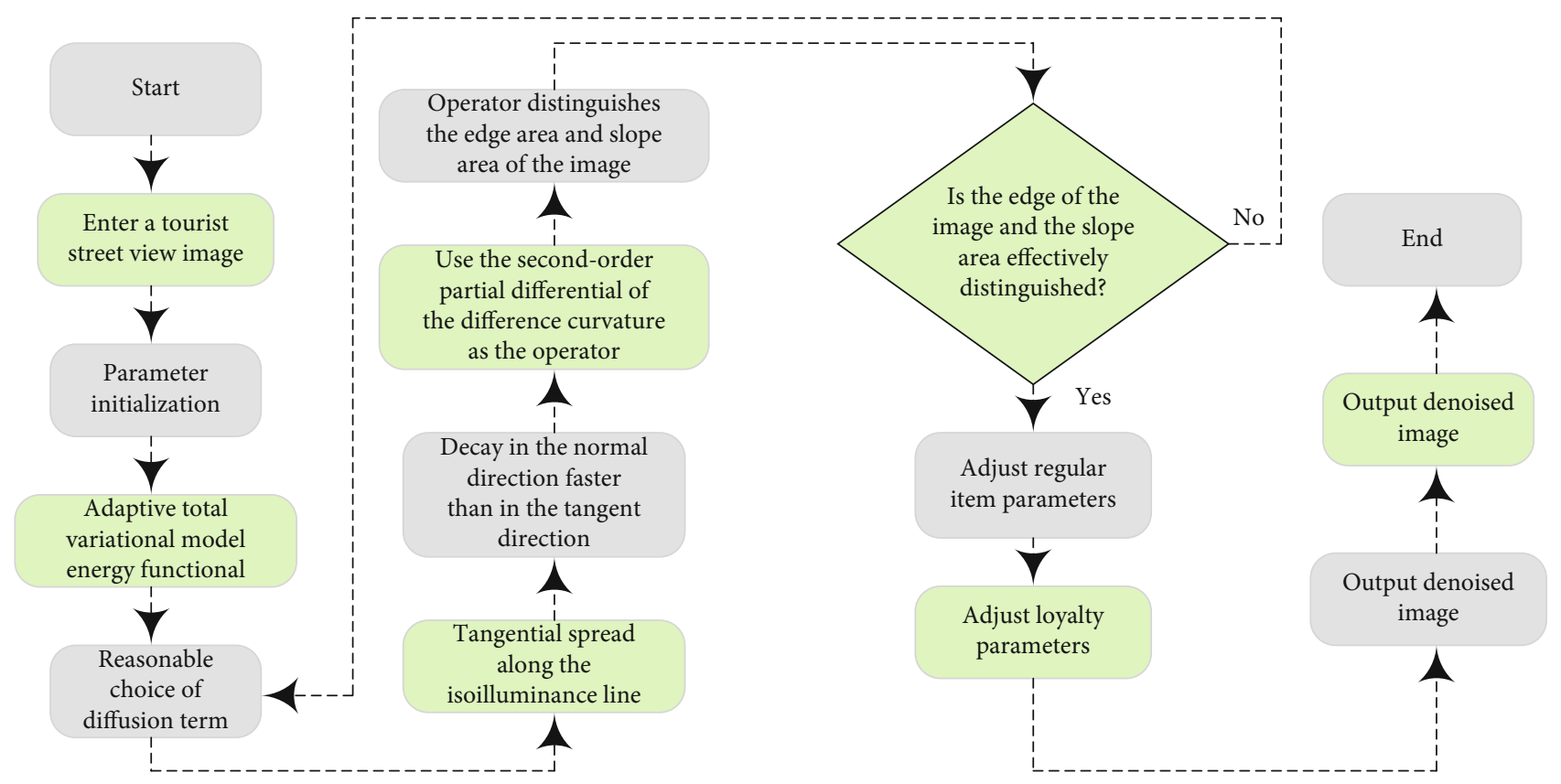

FIgURE 2: Self-adaptive full variational algorithm flow for second-order partial differential equations.

area of the tourist street view image. The diffusion makes the diffusion only proceed along the direction perpendicular to the gradient, thereby protecting the edge information such as the face contour and hair structure of the character.

Figures 3 and 4, respectively, show the comparison of the MSE and SSIM indicators of tourist street view images with a noise variance of 20 after processing the results of each model. It can be concluded that compared with other models, the mean square error of the tourist street view image denoising processing using the model in this paper is significantly reduced, and the structural similarity is greatly improved.

5.2. Denoising Experiment of Tourist Street Scene Image Combined with Improved PM and Recursive Filtering. Also, we use Matlab software for experimental simulation to verify the rationality and feasibility of the model algorithm in this paper, and evaluate the performance and benefit of the model through the same indicators as the previous article. The experiment selects the tourist street view image with a pixel size of $1024 \times 512$. We add Gaussian white noise with a variance of 40 to it and then perform experimental simulation analysis.

The PM model, YK model, TV model, WHT model, and the model in this paper are used to denoise the noise-added tourist street view images. We use Canny operator to extract edge features of the denoised result image. The tourist street scene images processed by the model in this paper are more effective in removing noise and protecting edge information and texture details, because the model in this paper can retain not only more detailed information such as background buildings by reducing the number of iterations but also detailed information in the edge area. We suppress the diffusion in the gradient direction, so as to protect the edge structure features such as the camera bracket and the outline of the building.
Figures 5 and 6, respectively, show the comparison of the MSE and SSIM indicators of tourist street view images with a noise variance of 40 after processing the results of each model. It can be concluded that, compared with the results obtained by other models, the mean square error of the tourist street scene image after denoising using the model in this paper is greatly reduced, and the structural similarity is further improved.

Figure 7 shows the comparison of the PSNR values of the noise reduction results of various models with different noise variances. It can be seen that as the noise variance increases, the model in this paper always maintains the highest peak signal-to-noise ratio, and the PSNR value has been above 0.92 . However, the peak signal-to-noise ratio of other models has not exceeded the value of the peak signal-to-noise ratio of the model in this paper. This proves that the model in this paper has more advantages than other models in the effectiveness and stability of denoising. It can be seen that the model in this paper has a higher peak signal-to-noise ratio than other models, which also shows that the model in this paper has better denoising ability.

5.3. Comparison of Model Performance Benefits. In order to further study the practicability of the model proposed in this paper, the campus scene view with special texture is selected and the image of tourist street scene is simulated by Matlab software. After adding Gaussian white noise with a variance of 60 , the simulation analysis is carried out.

We use PM model, YK model, TV model, WHT model, and the model of this article for denoising and keeping the time step of each model the same.

Table 1 shows the comparison of the MSE, PSNR, and SSIM indicators of the view tourist street view image with a noise variance of 60 after processing the results using each model. Analyzing the data in Table 1, it can be concluded that 


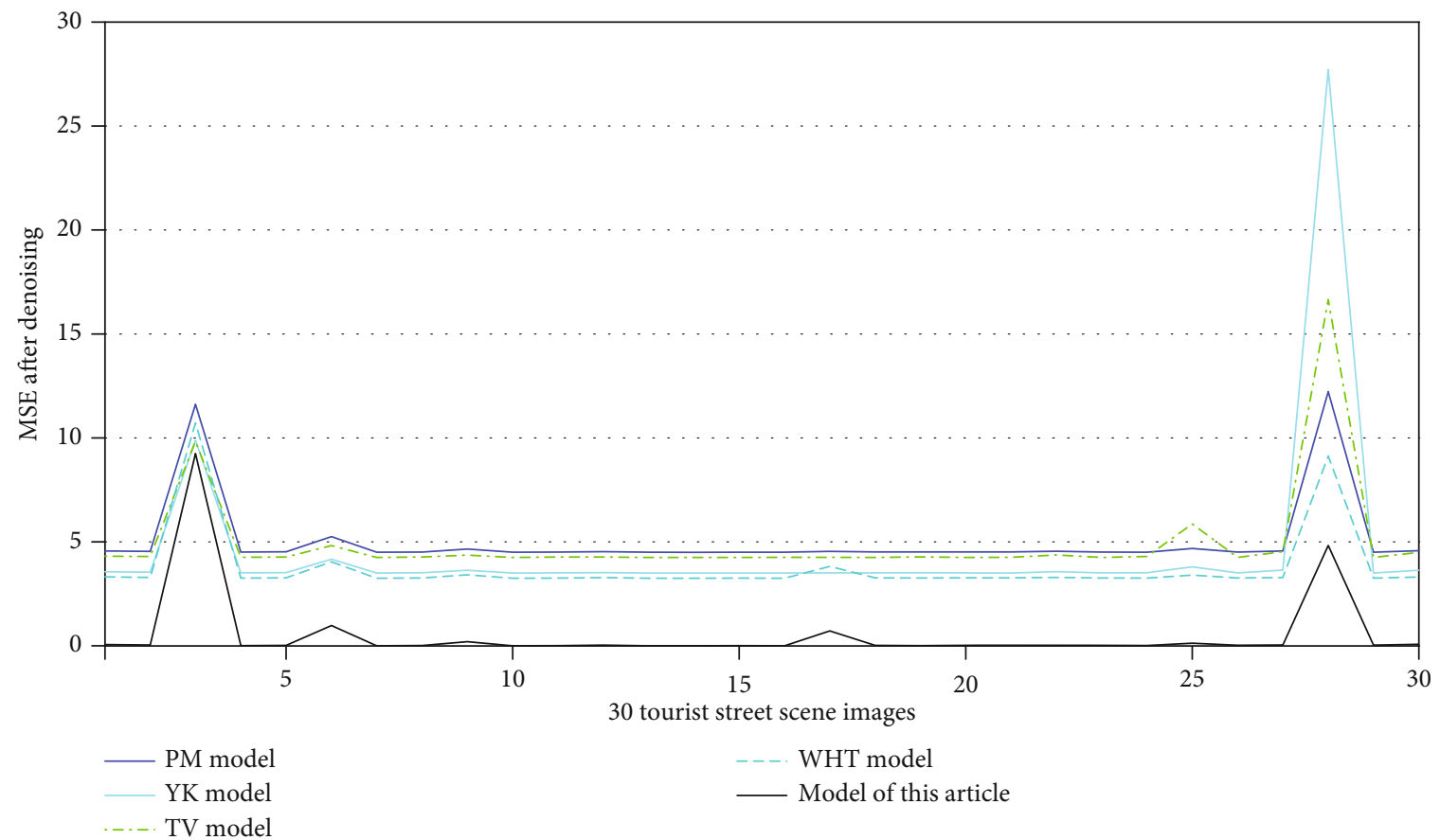

FIGURE 3: MSE comparison of tourist street view images with a noise variance of 20 after denoising using various models.

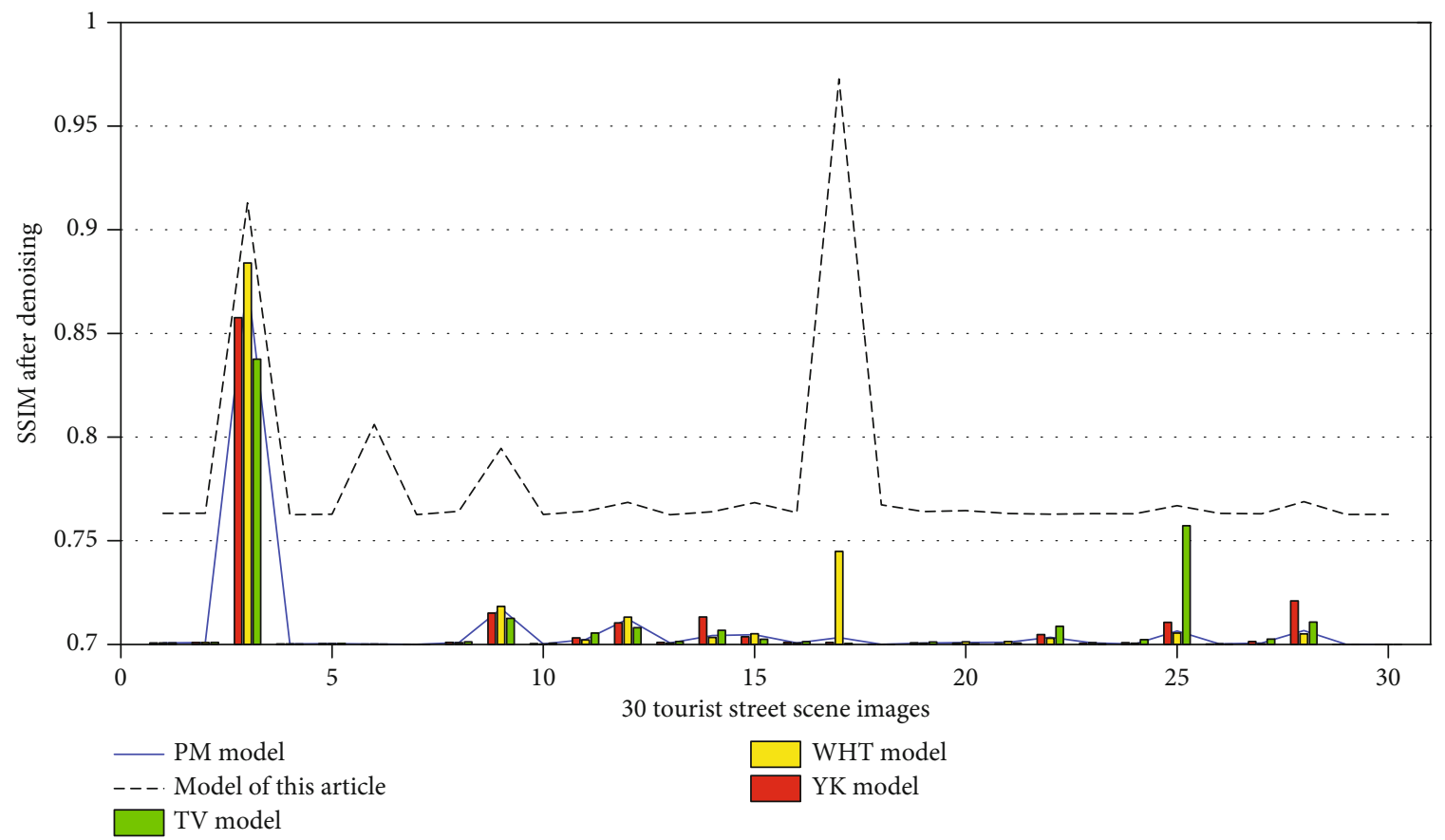

FIGURE 4: SSIM comparison of tourist street view images with a noise variance of 20 after denoising using various models. 


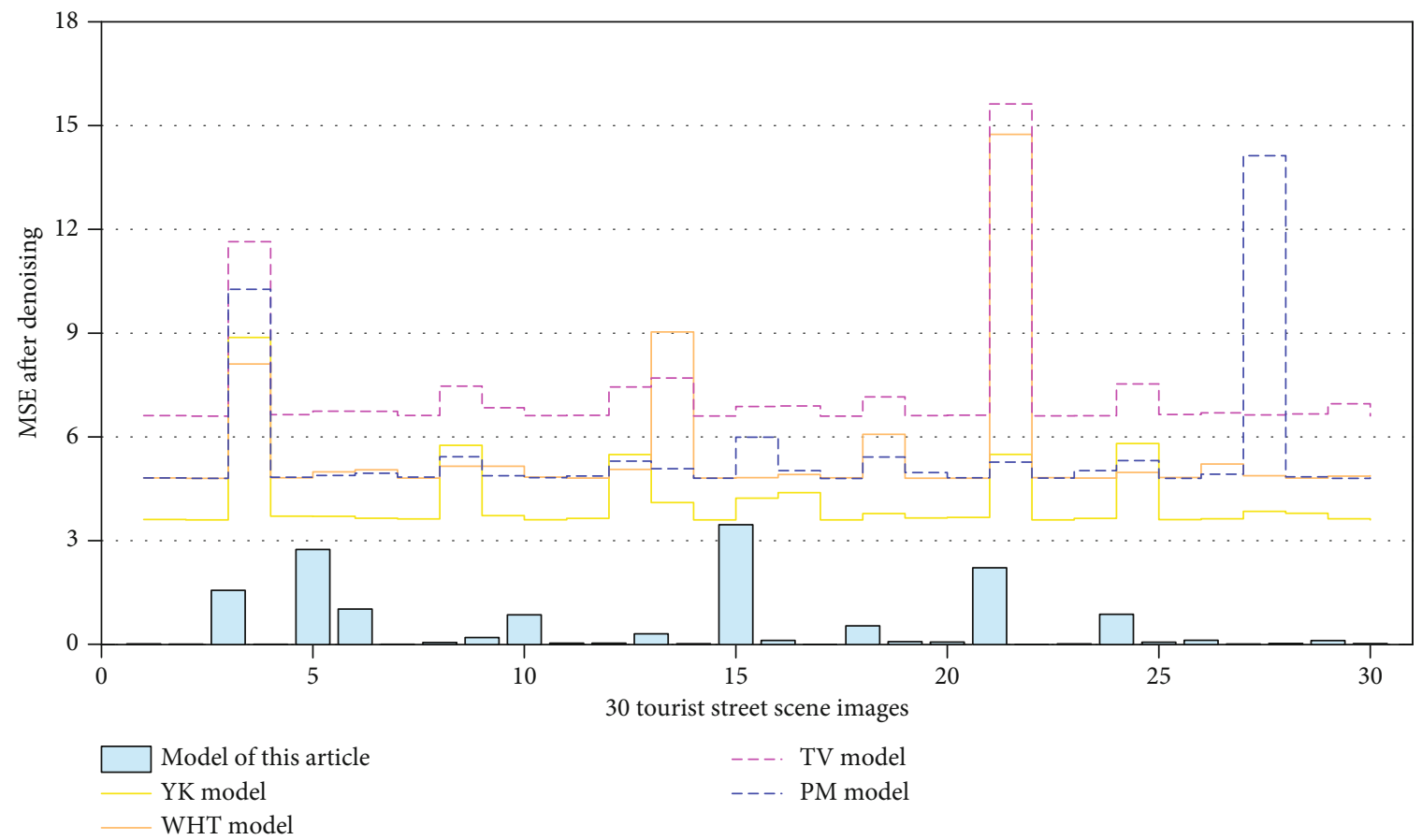

FIGURE 5: MSE comparison of tourist street view images with noise variance of 40 after denoising using various models.

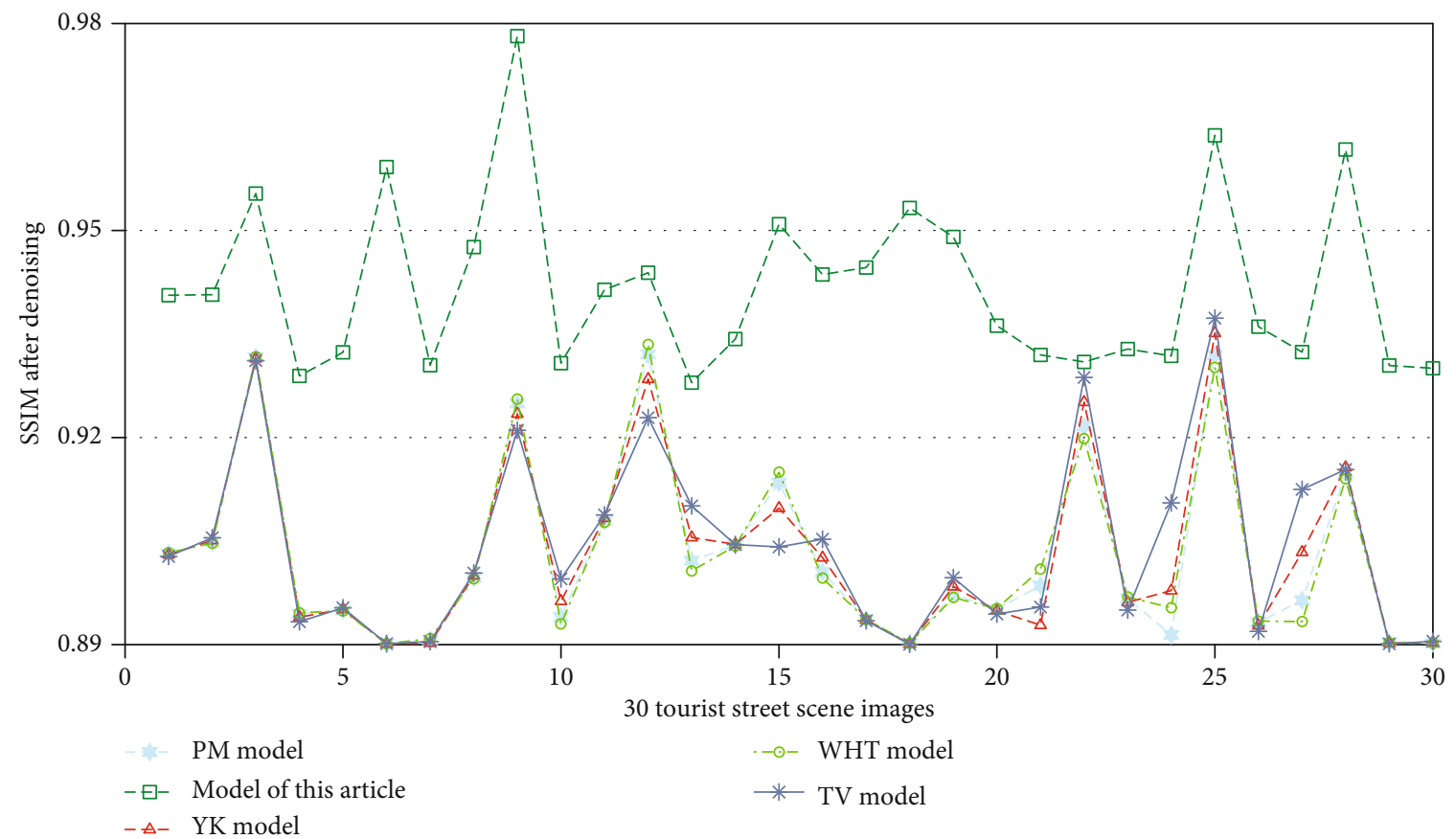

FIGURE 6: SSIM comparison of tourist street view images with noise variance of 40 after denoising using various models. 


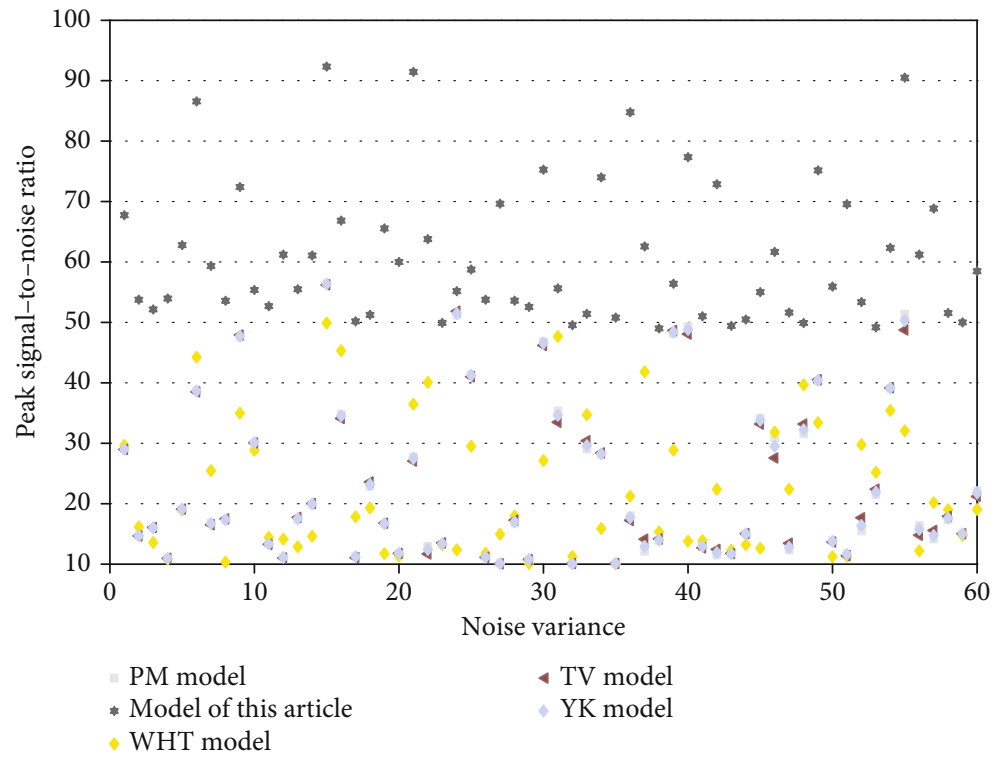

FIGURE 7: The peak signal-to-noise ratio of the denoising results of each model under different noise variances.

TABLE 1: Comparison of MSE, PSNR, and SSIM of view tourist street view images with noise variance of 60 after denoising by each model.

\begin{tabular}{ccccccc}
\hline & & $\begin{array}{c}\text { Model of this } \\
\text { article }\end{array}$ & $\begin{array}{c}\text { YK } \\
\text { model }\end{array}$ & $\begin{array}{c}\text { WHT } \\
\text { model }\end{array}$ & $\begin{array}{c}\text { PM } \\
\text { model }\end{array}$ & $\begin{array}{c}\text { TV } \\
\text { model }\end{array}$ \\
\hline \multirow{2}{*}{ View } & MSE & 4.21 & 13.02 & 18.03 & 10.01 & 22.12 \\
& PSNR & 47.03 & 21.02 & 28.04 & 34.09 & 19.02 \\
& SSIM & 0.98 & 0.69 & 0.87 & 0.78 & 0.82 \\
\hline
\end{tabular}

compared with the previous model, the mean square error of the tourist street view image obtained after the denoising of the model in this paper has been greatly reduced, and the peak signal-to-noise ratio and structural similarity have been significantly improved. This fully proves that the model proposed in this paper has better denoising performance than other models and shows that the model in this paper has the best denoising performance.

\section{Conclusion}

This paper discusses the basic knowledge of the variational method and the preliminary theorem of the variational basis used in the process of processing tourist street scene images with variational partial differential equations and explores the variational process of functional extremum. In order to solve the Euler-Lagrange equation in detail, this paper also gives the famous gradient descent flow method. Based on the classic variational PDE denoising model, this paper has carried out a detailed analysis, obtained a unified variational PDE energy functional model, also gave a detailed analysis of the diffusion performance of the total variation model, and then proposed an adaptive diffusion model of total variation. By improving the parameters of the loyalty term and regular term of the ROF model, the adaptive total variation denoising model of this paper is established. Through a large number of numerical experiments, it can be known that the improved adaptive total variation model has the advantages of better denoising effect and faster execution speed compared with the traditional total variation model. In the flat area of the tourist street view image, we adaptively use high-order total variation method to suppress the step effect of the tourist street view image in the process of removing noise and use the classic total variation method to remove noise in the edge area of the tourist street view image. From the experimental results, compared with the traditional partial differential equation denoising method, the model proposed in this paper has better denoising performance and stronger edge retention ability. The partial differential equations studied in this paper all appear in an integer order, and the application of fractional calculus in tourist street scene image processing has also received extensive attention, especially in tourist street scene image denoising. The current research results show the advantages and effects of fractional calculus in these applications. In addition, fractional calculus has made some research results in fractional dynamic systems, fractional chaotic systems, and fractional control systems. Although fractional calculus has achieved some research results in the field of tourist street scene image processing, the application research of fractional calculus in tourist street scene image processing is still in its infancy. Therefore, we are exploring how to use the tool of fractional calculus to deal with us, which is a future research project.

\section{Data Availability}

The data used to support the findings of this study are available from the corresponding author upon request.

\section{Conflicts of Interest}

We declare that there is no conflict of interest. 


\section{References}

[1] S. Li and Z. Zhi, "A novel nonlinear second order hyperbolic partial differential equation-based image restoration algorithm with directional diffusion," IEEE Access, vol. 8, pp. 131021131031, 2020.

[2] F. K. Golbaghi, M. Rezghi, and M. R. Eslahchi, "A hybrid image denoising method based on integer and fractionalorder total variation," Iranian Journal of Science and Technology, Transactions A: Science, vol. 44, no. 6, pp. 1803-1814, 2020.

[3] T. Barbu, "Detail-preserving fourth-order nonlinear PDEbased image restoration framework," Journal of Image and Graphics, vol. 8, no. 1, pp. 5-8, 2020.

[4] Y. Zhai, Z. Pan, and W. Wei, "Two variational models for image denoising using Jacobian of normals," IEEE Access, vol. 9, pp. 43301-43315, 2021.

[5] N. Mohamadi, A. R. Soheili, and F. Toutounian, "A new feature descriptor for image denoising," Iranian Journal of Science and Technology, Transactions A: Science, vol. 44, no. 6, pp. 1695-1700, 2020.

[6] D. Wang, J. J. Nieto, X. Li, and Y. Li, “A spatially adaptive edge-preserving denoising method based on fractional-order variational PDEs," IEEE Access, vol. 8, pp. 163115-163128, 2020.

[7] J. Bai and X. C. Feng, "Image decomposition and denoising using fractional-order partial differential equations," IET Image Processing, vol. 14, no. 14, pp. 3471-3480, 2020.

[8] X. Pan, Q. Zhao, and J. Liu, "Edge extraction and reconstruction of terahertz image using simulation evolutionary with the symmetric fourth order partial differential equation," Optoelectronics Letters, vol. 17, no. 3, pp. 187-192, 2021.

[9] T. Barbu, "Nonlinear PDE-based models for photon-limited image restoration," Applied Sciences, vol. 23, pp. 18-29, 2021.

[10] Y. Gu, "Finite element numerical approximation for two image denoising models," Circuits, Systems, and Signal Processing, vol. 39, no. 4, pp. 2042-2064, 2020.

[11] F. Kazemi Golbaghi, M. R. Eslahchi, and M. Rezghi, "Image denoising by a novel variable-order total fractional variation model," Mathematical Methods in the Applied Sciences, vol. 44, no. 8, pp. 7250-7261, 2021.

[12] L. I. U. Pei, J. I. A. Jian, and C. Li, "Image denoising algorithm based on boosting high order non-convex total variation model," Journal of Systems Engineering and Electronics, vol. 42, no. 3, pp. 557-567, 2020.

[13] G. Dong, M. Hintermueller, and Y. Zhang, "A class of secondorder geometric quasilinear hyperbolic PDEs and their application in imaging," SIAM Journal on Imaging Sciences, vol. 14, no. 2, pp. 645-688, 2021.

[14] L. Xu, G. Huang, Q.-l. Chen, H.-y. Qin, T. Men, and Y.-f. Pu, "An improved method for image denoising based on fractional-order integration," Frontiers of Information Technology \& Electronic Engineering, vol. 21, no. 10, pp. 14851493, 2020.

[15] H. Liang and J. Zhang, "Dual algorithm for truncated fractional variation based image denoising," International Journal of Computer Mathematics, vol. 97, no. 9, pp. 1849-1859, 2020.

[16] M. Nachaoui, "Parameter learning for combined first and second order total variation for image reconstruction," Advanced Mathematical Models \& Applications, vol. 5, no. 1, pp. 53-69, 2020.
[17] Y. Chen and T. He, "Image denoising via an adaptive weighted anisotropic diffusion," Multidimensional Systems and Signal Processing, vol. 32, no. 2, pp. 651-669, 2021.

[18] J. Yu, L. Chen, S. Zhou, L. Wang, H. Li, and S. Huang, “Adaptive image denoising for speckle noise images based on fuzzy logic," International Journal of Imaging Systems and Technology, vol. 30, no. 4, pp. 1132-1142, 2020.

[19] A. A. Yahya, J. Tan, B. Su et al., "BM3D image denoising algorithm based on an adaptive filtering," Multimedia Tools and Applications, vol. 79, no. 27-28, pp. 20391-20427, 2020.

[20] M. R. Rejeesh and P. Thejaswini, "MOTF: multi-objective optimal trilateral filtering based partial moving frame algorithm for image denoising," Multimedia Tools and Applications, vol. 79, no. 37-38, pp. 28411-28430, 2020.

[21] A. Theljani and Z. Belhachmi, "A discrete approximation of Blake and Zisserman energy in image denoising with optimal choice of regularization parameters," Mathematical Methods in the Applied Sciences, vol. 44, no. 5, pp. 3857-3871, 2021.

[22] H. Houichet, A. Theljani, and M. Moakher, "A nonlinear fourth-order PDE for image denoising in Sobolev spaces with variable exponents and its numerical algorithm," Computational and Applied Mathematics, vol. 40, no. 3, pp. 1-29, 2021.

[23] S. Zhang, Y. Yang, Q. Qin, L. Feng, and L. Jiao, "Rapid blind denoising method for grating fringe images based on noise level estimation," IEEE Sensors Journal, vol. 21, no. 6, pp. 8150-8160, 2021.

[24] S. Kumar, N. Kumar, and K. Alam, "A nonlinear anisotropic diffusion equation for image restoration with forwardbackward diffusivities," Recent Advances in Electrical \& Electronic Engineering (Formerly Recent Patents on Electrical \& Electronic Engineering), vol. 14, no. 4, pp. 428-434, 2021.

[25] T. Adam and R. Paramesran, "Hybrid non-convex secondorder total variation with applications to non-blind image deblurring," Signal, Image and Video Processing, vol. 14, no. 1, pp. 115-123, 2020. 